\title{
Influence of smooth muscle contractility by inhibition of crac channels activity
}

\author{
${ }^{1}$ Comenius University in Bratislava, \\ Jessenius Faculty of Medicine in Martin, \\ Biomedical Center Martin, Martin, Slovak Republic \\ ${ }^{2}$ Comenius University in Bratislava, \\ Jessenius Faculty of Medicine in Martin, \\ Department of Pharmacology, \\ Martin, Slovak Republic
}

Received 14 December, 2016, accepted 20 January, 2017

Abstract The present in vitro study was focused on the differences in expression and activity of calcium release-activated calcium (CRAC) channels of human term-pregnant and non-pregnant myometrium. The expression of Orai1 protein, as a functional subunit of CRAC channel, was significantly higher than in non-pregnant myometrium. Lower Orai1 protein expression did not influence the amplitude of contractile response of term-pregnant myometrium, but higher Orai1 expression observed in non-pregnant myometrium was related to the different influence of CRAC blocker on contraction frequency.

Keywords Oral N-acetylcysteine-Inhaled N-acetylcysteine-mucus clearance-airway reactivity

\section{INTRODUCTION}

Calcium ions $(\mathrm{Ca} 2+)$ play a substantial mediatory and regulatory role in nearly all cell activities. 3 types of ion channels are responsible for $\mathrm{Ca} 2+$ metabolism: receptoroperated calcium channels, voltage-gated calcium channels (VGCC) and store-operated calcium channels (Wray et al., 2001)).

VGCC is the principal mechanism of $\mathrm{Ca} 2+$ influx in spontaneously active myometrial tissue. The VGCC family L-type plays a vital role in the generation of phasic contractility of the uterus. Myometrial contractility depends on increase in intracellular $\mathrm{Ca} 2+$ resulting from the transmembrane influx of $\mathrm{Ca} 2+$ and the release of $\mathrm{Ca} 2+$ from endoplasmic reticulum (ER) (Noble et al., 2009). The main representative of store-operated channels is CRAC channel, which exhibits low conductance, strong inward rectification and remarkable Ca2+ selectivity (Sutovska et al., 2013; Parekh 2010). The CRAC channel is composed of two main elements: Orai1 channel protein of the plasma membrane and stromal interaction molecule 1 (STIM1) protein of ER (Hoth and Penner, 1992;
Potier and Trebak, 2008). A decrease of Ca2+ concentration in the ER triggers changes in STIM1 conformation, the sensor of $\mathrm{Ca} 2+$ concentration in the ER. Opening the Orai1 channels causes an influx of $\mathrm{Ca} 2+$ into the cytoplasm of the cell. The rising concentration of $\mathrm{Ca} 2+$ in the cytoplasm induces closing of the channel by Ca2+-dependent inactivation (Derler et al., 2012).

Recently, the expression of CRAC channels in smooth muscle (SM), including the myometrium, has been confirmed $(7,8)$. Therefore, this in vitro experimental study aimed to specify the role of CRAC channels in the contractility of human termpregnant and non-pregnant myometrium.

\section{MATERIAL AND METHODS}

All processes were approved by the Institutional Ethics Committee of the Jessenius Faculty of Medicine, (decision No. EK 1124/2012). Patient recruitment was conducted by the provision of information sheets, and written informed 
Table 1. CRAC blocker influence on frequency (per hour) and amplitude of contraction ( $\mathrm{mN}$ ) of pregnant and non-pregnant myometrium. All data are expressed as average \pm SEM. Results with $P<0.05$ were considered statistically significant. $P$ vs. Oxy.

\begin{tabular}{|c|c|c|c|c|}
\hline & \multicolumn{2}{|c|}{ Non-pregnant $\mathbf{n = 7}$} & \multicolumn{2}{c|}{ Pregnant $\mathbf{n = 6}$} \\
\hline & Frequency & Amplitude of contraction & Frequency & Amplitude of contraction \\
\hline Oxy $(1 \mu \mathrm{M})$ & $41.2 \pm 2.9$ & $1.5 \pm 0.2$ & $48.3 \pm 4.0$ & $2.3 \pm 0.2$ \\
\hline Sal $(100 \mu \mathrm{M})$ & $28.3 \pm 3.2 *$ & $0.9 \pm 0.1 *$ & $28 \pm 2.3^{* *}$ & $1.3 \pm 0.2 * *$ \\
\hline CRAC $(1 \mu \mathrm{M})$ & $20.3 \pm 3.3^{* *}$ & $1.1 \pm 0.1$ & $34.7 \pm 3.1 *$ & $2.1 \pm 0.1$ \\
\hline CRAC $(10 \mu \mathrm{M})$ & $26.3 \pm 2.6 *$ & $1 \pm 0.1 *$ & $33.7 \pm 4.4^{* *}$ & $2 \pm 0.1 *$ \\
\hline CRAC $(100 \mu \mathrm{M})$ & $29.3 \pm 2.5$ & $0.9 \pm 0.1 *$ & $27.5 \pm 5.8^{* *}$ & $1.9 \pm 0.1 *$ \\
\hline
\end{tabular}

consent was obtained. Samples of myometrium were collected in cooperation with the Clinic of Gynaecology and Obstetrics. Human myometrium originated from woman without any underlying conditions and undergoing elective caesarean section at term (week of pregnancy 38.7 \pm 0.7 ) and non-pregnant myometrium of women undergoing total hysterectomy (aged $47 \pm 2.24$ ) due to uterine myoma from macroscopic healthy tissue.

Uterus SM reactivity was verified using the organ tissue bath methodology (Franova et al., 2009). Myometrium samples were incubated for one hour in Krebs-Henzeleit solution. Oxytocin was applied directly into the chamber to induce SM contractions. Cumulative doses $(1 \mu \mathrm{M}, 10 \mu \mathrm{M}, 100 \mu \mathrm{M})$ of tested substance CRAC channel blocker 3-fluoropiridine-4carboxylic acid (FPCA) and salbutamol $(100 \mu \mathrm{M})$ were applied into the chamber after 5 minutes from oxytocin application. The amplitude and frequency of SM contraction was recorded.

The modulator of CRAC channels activity FPCA was purchased from TOCRIS (USA). Oxytocin and salbutamol were obtained from Sigma Aldrich SR. All substances were dissolved in aqua pro injection.

Orai1 protein expression, a pore-forming subunit of the CRAC channel, was determined by immunohistochemistry. The reaction was visualized using the LSAB visualization kit and diaminobenzidine chromogen. The Orai1 expression was evaluated using a rating scale, where 1 stood for no degree of positivity, 2 mild or sporadic positivity and level 3 distinct positivity.

Fisher's exact test was selected to evaluate the IHC features and amplitude of contraction was evaluated using one-way ANOVA with Bonferroni post-hoc test.

\section{RESULTS}

Uterus SM contractions, induced by oxytocin, were reduced after FPCA application. The amplitude of contractions was significantly reduced after the addition FPCA at 10 and 100 $\mu \mathrm{M}$ concentration in both groups (Tab. 1). The FPCA ability to reduce oxytocin-induced contraction were compared to

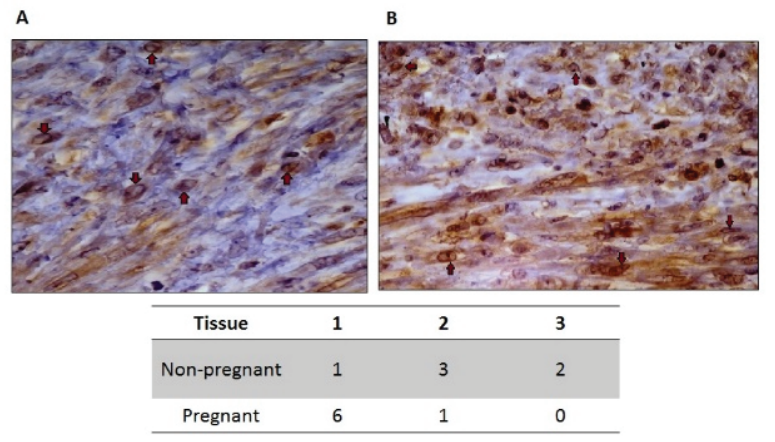

Figure 1. The immunohistochemical analysis of A) pregnant and B) non-pregnant myometrium. Cells with distinct plasma membrane Orai 1 positivity are marked by red arrows. 1 - no degree of positivity, 2 - mild positivity, 3 -distinct positivity.

clinically used drug salbutamol. FPCA effect in samples from non-pregnant women was comparable with salbutamol, but in samples from pregnant group, salbutamol was more effective than FPCA (Tab. 1). The frequency of uterine contractions from pregnant group reduced significantly after the application of CRAC channel blocker. The higher concentration of the tested substance led to an increase in the contraction frequency (Tab. 1). FPCA reduced the contraction frequency of myometrium from patients with myoma, which was almost comparable to the reference drug (Tab. 1).

Higher expression of Orai1 protein was in samples from nonpregnant group than in samples of pregnant myometrium (Fig. 1).

\section{DISCUSSION}

Recently, the expression of CRAC channels in smooth muscle, including the myometrium, has been confirmed in several studies. These studies confirmed the presence of CRAC channels not only in smooth muscle of myometrium but also in smooth muscle of respiratory tract and others (Sutovska et al. 2013; Potier and Trebak, 2008; Derler et al. 2012). The present study not only confirmed the expression of Orai1 
protein, the main pore-forming subunit of CRAC channels in myometrium, but for the first time also distinguished distinct changes between Orai1 protein expression in non-pregnant myometrium and in term-pregnant myometrium.

Functional part of this experimental study aimed to specify the role of CRAC channels in the contraction/relaxation of human term-pregnant and non-pregnant myometrium. Data presented in this paper provide strong evidence of CRAC channel's involvement in the contracting activity of myometrium. Moreover, lower expression of Orai1 protein did not influence the amplitude of the contractile response of term-pregnant myometrium on cumulative doses of CRAC blocker, but higher expression of Orai1 in non-pregnant myometrium was very probably related to different influence of this agent on frequency of contraction.

\section{ACKNOWLEDGEMENT}

The authors acknowledge Katarina Jesenska for her outstanding technical assistance, Marian Adamkov for immunohistochemical analysis and Karol Dokus for obtaining specimens. This study was supported by the Centre of Experimental and Clinical Respirology I, Centre of Experimental and Clinical Respirology II, BioMed and 'The increasing of opportunities for career growth in research and development in the medical sciences' projects co-financed from EC sources, grant MZ 2012/35-UKMA-12, APVV-0305-12 and grant VEGA no. 1/0127/13.

\section{References}

[1] Derler I, Madl J, Schütz G, Romanin CH. Structure, regulation and biophysics of I(CRAC), STIM/Orai1. Adv Exp Med Biol 2012; 740: 383-410.

[2] Franova S, Janicek F, Visnovsky J, Dokus K, Zubor P, Sutovska M, Nosalova G. Utero-relaxant effect of PDE4-selective inhibitor alone and in simultaneous administration with $\beta 2$-mimetic on oxytocin-induced contractions in pregnant myometrium. Int J Gynaecol Obstet. 2009; 35: 20-25.

[3] Hoth M, Penner R. Depletion of intracellular calcium stores activates a calcium current in mast cells. Nature 1992; 355: 353-356.

[4] Noble K, Matthew A, Burdyga T, Wray S. A review of recent insights into the role of the sarcoplasmic reticulum and Ca entry in uterine smooth muscle. Eur J Obstet Gynecol Reprod Biol 2009; 144 (1 suppl): S11-S19.
[5] Parekh AB. Store-operated CRAC channels: Function in health and disease. Nat Rev Drug Discov 2010; 9: 399-410.

[6] Potier M, Trebak M. New developments in the signalling mechanisms of the store-operated calcium entry pathway. Pflugers Arch 2008; 457: 405-415.

[7] Sutovska M, Kocmalova M, Adamkov M, Vybohova D, Mikolka P, Mokra D, Hatok J, Antosova M, Franova S. The long-term administration of Orai 1 antagonist possesses antitussive, bronchodilatory and anti-inflammatory affects in experimental asthma model. Gen. Physiol. Biophys.2013; 32:251-259

[8] Wray S, Kupittayanant S, Shmygol A, Smith RD, Burdyga T. The physiological basis of uterine contractility: A short review. Exp Physiol 2001; 86: 239-246. 